\title{
Correction: Guanidinylated cationic nanoparticles as robust protein antigen delivery systems and adjuvants for promoting antigen-specific immune responses in vivo
}

Cite this: J. Mater. Chem. B, 2016, 4, 6746

\author{
Pan Li, ${ }^{a}$ Gaona Shi, ${ }^{a}$ Xiuyuan Zhang, ${ }^{a}$ Huijuan Song, ${ }^{a}$ Chuangnian Zhang, ${ }^{a}$ \\ Weiwei Wang, ${ }^{* a}$ Chen $\mathrm{Li}^{\text {a }}$ Bing Song, ${ }^{\mathrm{b}}$ Chun Wang ${ }^{* \mathrm{ac}}$ and Deling Kong ${ }^{\mathrm{a}}$
}

DOI: 10.1039/c6tb90140a

Correction for 'Guanidinylated cationic nanoparticles as robust protein antigen delivery systems and adjuvants for promoting antigen-specific immune responses in vivo' by Pan Li et al., J. Mater. Chem. B,

www.rsc.org/MaterialsB

2016, 4, 5608-5620.

In this article, several fluorescence images in Fig. 6 were mixed up between two groups. Specifically, in Fig. 6A, during live imaging experiment, after data acquisition, the images of the OVA group/day 4 of varying fluorescence intensity were mistakenly labeled as images of the Nano.-OVA group/day 4, 8, 10. For the same reason, in Fig. 6B, the $6 \mathrm{~h}$ images of the Nano.-OVA and OVA groups were actually different images of the same mouse but mistakenly labeled as such. It was likely that these mistakes happened during transferring and renaming image files from the live imaging facility back to the lab. As a result, the original correct image files have been lost.

To correct these mistakes, this experiment was repeated and correct images of mice were recaptured. This experiment was performed in accordance with the previous one, including the antigen dose, injection location, sampling time, animal numbers as well as the exposure time during imaging. The newly obtained correct images and quantification result of fluorescence signals are shown in this correction notice. No corrections are required to the text of the article, including the results analysis and the conclusion. The authors sincerely apologize for any inconvenience this may have caused.
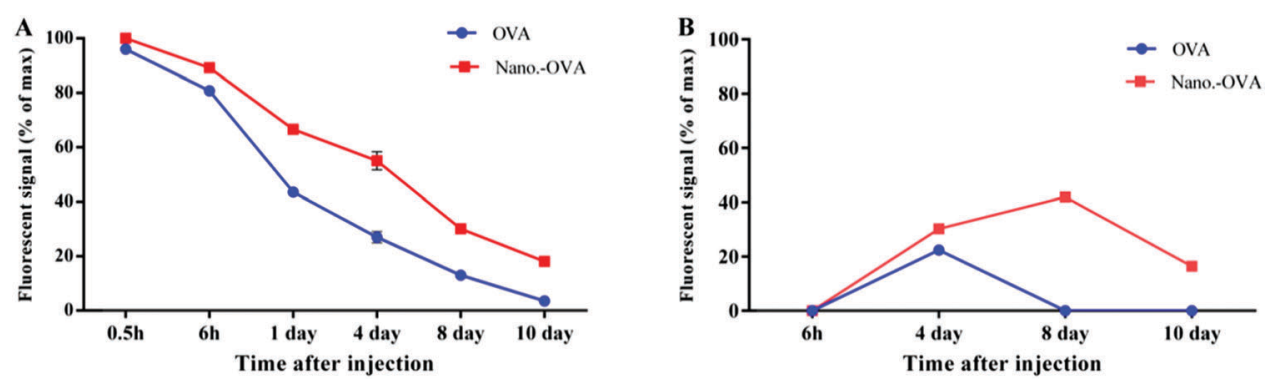

Fig. S6

\footnotetext{
${ }^{a}$ Tianjin Key Laboratory of Biomaterial Research, Institute of Biomedical Engineering, Chinese Academy of Medical Sciences and Peking Union Medical College, Tianjin 300192, China. E-mail: wwwangtj@163.com

${ }^{b}$ Cardiff Institute of Tissue Engineering \& Repair, School of Dentistry, College of Biomedical and Life Sciences, Cardiff University, UK

${ }^{c}$ Department of Biomedical Engineering, University of Minnesota, Minneapolis, Minnesota, USA. E-mail: wangx504@umn.edu
} 

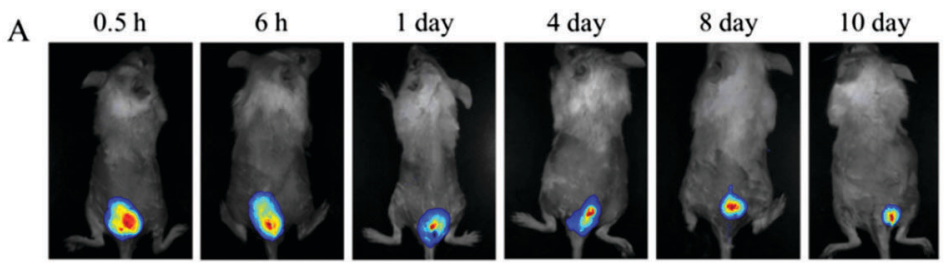

Nano.-OVA
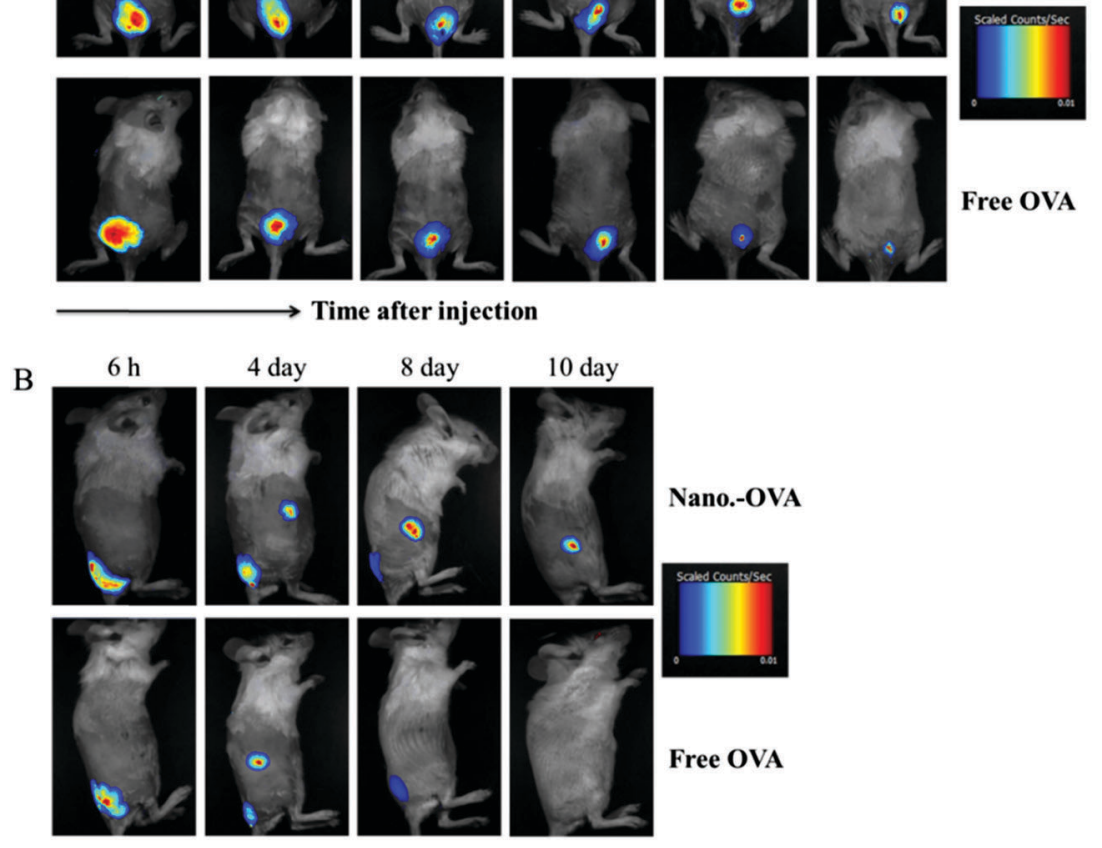

Time after injection

B

10 day

Time after injection

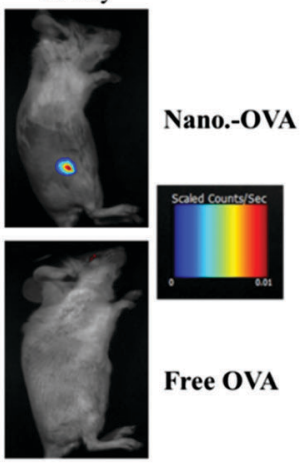

Fig. 6

The Royal Society of Chemistry apologises for these errors and any consequent inconvenience to authors and readers. 